\title{
UPPER CRITICAL MAGNETIC FIELD AND SPECIFIC HEAT STUDIES OF LaCu
}

\author{
J. W. Chen \\ Department of Physics, \\ National Taiwan University, Taipei, Taiwan, R. O. C. \\ and \\ Y. Y. Chen \\ Institute of Physics, Academia Sinica, Taipei, Taiwan, R. O. C.
}

(Received 25 February 1994 by P. Wachter)

\begin{abstract}
We have performed the upper critiical magnetic field $\mathrm{H}_{\mathrm{c} 2}(\mathrm{~T})$ and specific heat $\mathrm{C}(\mathrm{T})$ studies of $\mathrm{LaCu}$. The initial slope of $\mathrm{H}_{\mathrm{c} 2}(\mathrm{~T})$ near the zero field superconducting transition temperature $\mathrm{T}_{\mathrm{c}}=6.15 \mathrm{~K}$ has a value of $2.97 \mathrm{kG} / \mathrm{K}$, from which a zero-temperature superconducting coherence length $\xi_{0} \approx 162 \AA$ can be inferred. Analysis using the specific heat data yields an effective mass of $4 \mathrm{~m}$ for the conduction electrons and a Debye temperature $\Theta_{D}=148 \mathrm{~K}$ for this compound.
\end{abstract}

\section{Introduction}

There have been a lot of research activities about the interaction between superconductivity and magnetism in the past few decades. Early experiments involved dissolving small amounts of magnetic ions into a superconducting matrix, such as $\mathrm{La}_{1-\mathrm{x}} \mathrm{Gd}_{\mathrm{x}} 1,2$ and $\left(\mathrm{La}_{1-\mathrm{x}} \mathrm{Gd}_{\mathrm{x}}\right) \mathrm{Al}_{2}{ }^{3}$, and demonstrated the destructive effect of the magnetic ions on the superconducting transition temperature $\mathrm{T}_{\mathrm{C}}$. Moreover, interesting phenomena between the magnetic ordering and superconductivity were observed in many systems. For example, in $\left(\mathrm{Ce}_{1-x} \mathrm{Ho}_{x}\right) \mathrm{Ru}_{2}{ }^{4-6}$, superconductivity was observed to coexist with short range magnetic ordering at $x$ $=0.27$; reentrant superconductivity was observed in $\left(\mathrm{La}_{1}\right.$ $\left.{ }_{x} G_{x}\right) R_{2}$ near $x=0.05$ wherein the sample becomes superconducting at a temperature $T_{1}$ and returns to the normal state at a lower temperature $\mathrm{T}_{2}$ at which short-range spin glass type of magnetic ordering forms in the sample?. Later discovery of the Chevrel phase compounds 8.9 and the $R \mathrm{Rh}_{4} \mathrm{~B}_{4}(\mathrm{R}=$ rare earth ions) compounds 10,11 has renewed the interest in the question between superconductivity and long range magnetic ordering because the $R$ ions in these systems occupy a periodic array in the lattice 12,13 . Among the large varieties of $\mathrm{R}-\mathrm{Cu}$ systems, $\mathrm{RCu}$ have been reported to crystallize in FeB-type structure for $\mathrm{R}=\mathrm{La}, \mathrm{Ce}, \mathrm{Pr}, \mathrm{Nd}$, and $\mathrm{Yb}^{14-16}$. An ac susceptibility study of $\mathrm{LaCu}$ indicates superconducting transition occurs at $\mathrm{T} \approx 5.85 \mathrm{~K}^{17}$. An antiferromagnetic transition was observed in $\mathrm{CeCu}$ with a Néel temperature $T_{N}$ ranging from $2.7 \mathrm{~K}$ to $3.6 \mathrm{~K}^{15,18}$. Magnetization measurements of $\mathrm{PrCu}$ and $\mathrm{NdCu}$ reveal anomalous characteristics in these compounds ${ }^{16}$. Recently we found that $\mathrm{NdCu}$ becomes antiferromagnetic with $\mathrm{T}_{\mathrm{N}} \approx$ $10 \mathrm{~K}$ and a weak antiferromagnetic transition occurs in $\mathrm{PrCu}$ at $T=13 \mathrm{~K}^{19}$. Therefore it is interesting to investigate the effect of various types of magnetic ordering on the superconductivity in the $\mathrm{La}_{1-x} \mathrm{R}_{x} \mathrm{Cu}(\mathrm{R}=\mathrm{Ce}, \mathrm{Pr}$, and $\mathrm{Nd})$ systems. Before doing this, it is necessary to have an understanding of the normal state and superconducting state properties of the superconducting matrix $\mathrm{LaCu}$. In this paper, we report the upper critical magnetic field $\mathrm{H}_{\mathrm{c} 2}(\mathrm{~T})$ and the specific heat $\mathrm{C}(\mathrm{T})$ studies of the $\mathrm{LaCu}$ compound.

\section{Experimental Details}

The polycrystalline samples of $\mathrm{LaCu}$ were prepared by arc-melting the high purity elements (La: $99.99 \%$, Cu: $99.999 \%$ ) together on a water cooled copper hearth in a $\mathrm{Zr}$ gettered argon atmosphere, with no subsequent annealing. The $\mathrm{H}_{\mathrm{c} 2}(\mathrm{~T})$ data in this study were determined from the ac electrical resistivity $p(T)$ measurements on a bar-shaped sample performed in a helium dewar using a four-wire resistance bridge operating at a frequency of $16 \mathrm{~Hz}$. A calibrated $\mathrm{Ge}$ thermometer was used for the zero field measurement and for the calibration of a carbon glass thermometer which was used as the thermometer for the non-zero field measurements up to $H=15 \mathrm{kG}$ in this study. Dc magnetization measurement was performed using Quantum Design MPMS SQUID magnetometer from $2 \mathrm{~K}$ to $10 \mathrm{~K}$ in an applied magnetic field of $10 \mathrm{G}$. The specific heat $\mathrm{C}(\mathrm{T})$ measurement was performed on a small $\mathrm{LaCu}$ sample in a $\mathrm{He}^{3}-\mathrm{He}^{4}$ dilution refrigerator using the relaxation method in the temperature range of $0.3 \mathrm{~K} \leq \mathrm{T} \leq 16 \mathrm{~K}$.

\section{Results and Discussion}

The ac electrical resistivity $\rho$ versus temperature $T$ curve in zero magnetic field for $\mathrm{LaCu}$ are plotted in Fig. 1(a) for 0 $\mathrm{K} \leq \mathrm{T} \leq 300 \mathrm{~K}$. Above the superconducting transition temperature, $\rho(T)$ curve exhibits typical characteristics of common metals and can be expressed in terms of $p(T)=p_{i}$ $+\rho_{\mathrm{ph}}(\mathrm{T})$ with a residue resistivity $\rho_{\mathrm{i}}=1.33 \mu \Omega-\mathrm{cm}$ and the phonon contribution to the resistivity $\rho_{\mathrm{ph}}(\mathrm{T})$ can be fitted by the semi-emperical Bloch-Grüneisen formula using a Debye temperature $\Theta_{D}=120 \mathrm{~K}$ and a electron-phonon interaction constant $C$ of $=0.25 \mu \Omega-\mathrm{cm} / \mathrm{K}$. The large value of the $R R$ ratio $(\rho(300 \mathrm{~K}) / \rho(7 \mathrm{~K})=57)$ reflects high sample quality. At $\mathrm{T} \approx 6.4 \mathrm{~K}$, the resistivity drops rapidly to zero and the sample becomes superconducting with the midpoint transition temperature $\mathrm{T}_{\mathrm{c}}(50 \%)=6.15 \mathrm{~K}$ and a transition 

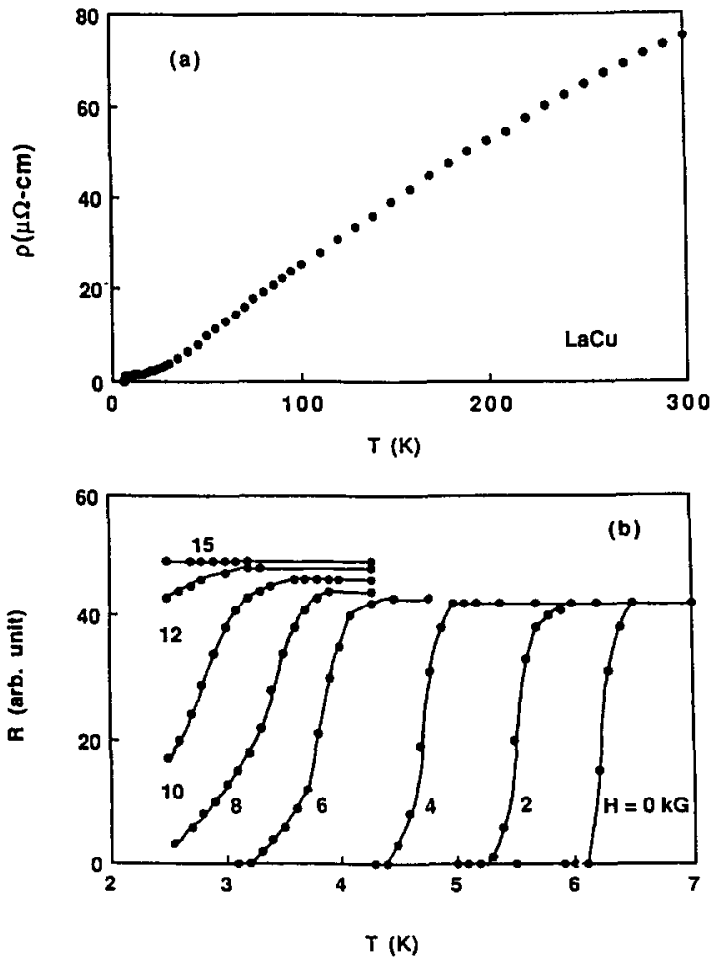

Fig. 1(a). The ac electrical resistivity $\rho$ as a function of temperature $\mathrm{T}$ in zero magnetic field for $\mathrm{LaCu}$. 1(b). Selective curves for the ac electrical resistance $R$ as a function of temperature $\mathrm{T}$ in different applied magnetic fields $\mathrm{H}$ below $15 \mathrm{kG}$.

width $\Delta \mathrm{T}_{\mathrm{c}}$ of $0.2 \mathrm{~K}$. Dc magnetization measurement at $\mathrm{H}=$ $10 \mathrm{G}$ reveals diamagnetic transition occurring at the same temperature and indicates bulk superconductivity in this sample. Shown in Fig. 1(b) are selected curves for ac electrical resistance $R$ as a function of temperature $T$ in different applied magnetic fields $H$ below $15 \mathrm{kG}$. The normal state resistivity increases slightly with increasing applied magnetic field $\mathrm{H}$ which becomes more pronounced for $\mathrm{H} \geq 6 \mathrm{kG}$. Moreover, with increasing magnetic field, the superconducting transition curves shift to lower temperature and the transition widths increase. Finally, when the field gets higher than $12 \mathrm{kG}$, the superconducting transition temperature is lower than $2.5 \mathrm{~K}$.

Shown in Fig. 2(a) are the heat capacity $C$ divided by temperature $\mathrm{T}$ vs $\mathrm{T}$ data for $\mathrm{LaCu}$. $\mathrm{A}$ jump in $\mathrm{C}$ is observed near $\mathrm{T}=5.8 \mathrm{~K}$, which is close to the value of $T_{c}$ observed in the ac electrical resistivity measurement. The observed value of $\Delta \mathrm{C} / \gamma \mathrm{T}_{\mathrm{c}}$ is about 0.25 , which is substantially smaller than 1.43 for the weak coupling superconductors. Nevertheless, this jump in $\mathrm{C}$ indicates bulk superconductivity in the sample. The $\mathrm{C}(\mathrm{T}) / \mathrm{T}$ vs $\mathrm{T}^{2}$ data for $\mathrm{LaCu}$ are displayed in Fig. 2(b). A marked departure from the linear relation above $T_{c}$ is observed which indicates the Debye temperature $\Theta_{D}$ for $\mathrm{LaCu}$ is quite low since the low temperature approximation for heat capacity $C=\gamma T+\beta T^{3}$ holds only for $T / \Theta_{D} \ll 1$. The zero temperature value of $\gamma$ is $\approx 10 \mathrm{~mJ} / \mathrm{mole}^{2} \mathrm{~K}^{2}$ and the value of $\beta$ as obtained from the slope at low temperatures is $1.18 \mathrm{~mJ} / \mathrm{mole}-\mathrm{K}^{4}$. From the value of $\beta$, the Debye
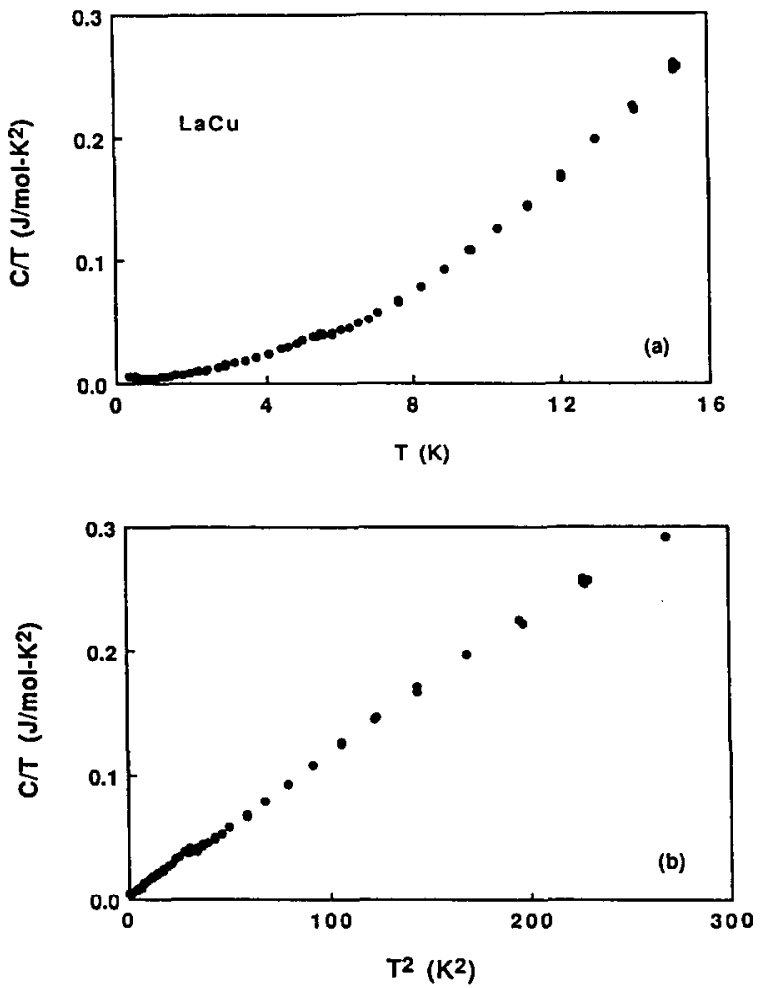

Fig. 2(a). The heat capacity $C$ divided by temperature $T$ vs $\mathrm{T}$ for $\mathrm{LaCu}$. 2(b). C(T)/T vs $\mathrm{T}^{2}$ data for $\mathrm{LaCu}$.

temperature can be calculated from the relation $\Theta_{D}=(1.946$ $\left.x 10^{6} \mathrm{~N} / \beta\right)^{1 / 3}$ with $\mathrm{N}$ being the number of atoms per formula unit ( $N=2$ here). The obtained value of $\Theta_{D}$ is $148 \mathrm{~K}$, which is close to the value of $\Theta_{D}$ deduced from the $\rho(T)$ fitting.

The upper critical magnetic field as a function of temperature $\mathrm{H}_{\mathrm{c} 2}(\mathrm{~T})$ for $\mathrm{LaCu}$ determined from the ac electrical resistivity measurements are plotted in Fig. 3 with the solid circles represent $T_{c}(50 \%)$ 's, the horizontal bars represent the transition widths and the arrows at high fields indicate the transition is not complete at $\mathrm{T} \approx 2.5 \mathrm{~K}$. The overall shape of the $\mathrm{H}_{\mathrm{c} 2}$ (T) curve is typical of a conventional type II superconductor. The observed value of $\mathrm{H}_{\mathrm{c} 2}(\mathrm{~T}=0$ $\mathrm{K})$ is $\approx 12.6 \mathrm{kG}$. which is an order of magnitude lower than the obtained value of the paramagnetic critical field $\mathrm{H}_{\mathrm{p}}\left(\mathrm{H}_{\mathrm{p}}=\right.$ $\left.18.4 \times \mathrm{T}_{\mathrm{c}}(\mathrm{H}=0) \mathrm{kG} \approx 113 \mathrm{kG}\right)$ and indicates that the orbital critical magnetic field $\mathrm{H}^{*}{ }_{\mathrm{c} 2}(\mathrm{~T})$ is mainly responsible for the $\mathrm{H}_{\mathrm{c} 2}(\mathrm{~T})$ in this sample. The linear portion of the $\mathrm{H}_{\mathrm{c} 2}$ vs $\mathrm{T}$ curve at low magnetic fields has a slope $\left(-\mathrm{dH}_{\mathrm{c}} / \mathrm{dT}\right) \mathrm{TcO}$ $=2.97 \mathrm{kG} / \mathrm{K}$ which can be used to calculate the zerotemperature orbital critical magnetic field $\mathrm{H}^{*}{ }_{\mathrm{c} 2}(0)$ using the weak-coupling formula ${ }^{20} \mathrm{H}_{\mathrm{c} 2}^{*}(0)=0.693 \times\left(-\mathrm{dH}_{\mathrm{c} 2} / \mathrm{dT}\right) \mathrm{Tc} \times$ $\mathrm{T}_{\mathrm{c} 0}$ which yields a value of $\approx 12.6 \mathrm{kG}$. This value can be used to estimate the superconducting coherence length $\xi_{0}$ at $\mathrm{T}=0 \mathrm{~K}$ through the relation ${ }^{21} \mathrm{H}^{*}{ }_{\mathrm{c} 2}(0)=\Phi_{0} / 2 \pi \xi_{0}{ }^{2}$, where $\Phi_{0}=\mathrm{ch} / 2 \mathrm{e}=2.07 \times 10^{-7} \mathrm{G}-\mathrm{cm}^{2}$ is the flux quantum. The obtained value of $\xi_{0}$ is $\approx 162 \AA$. 


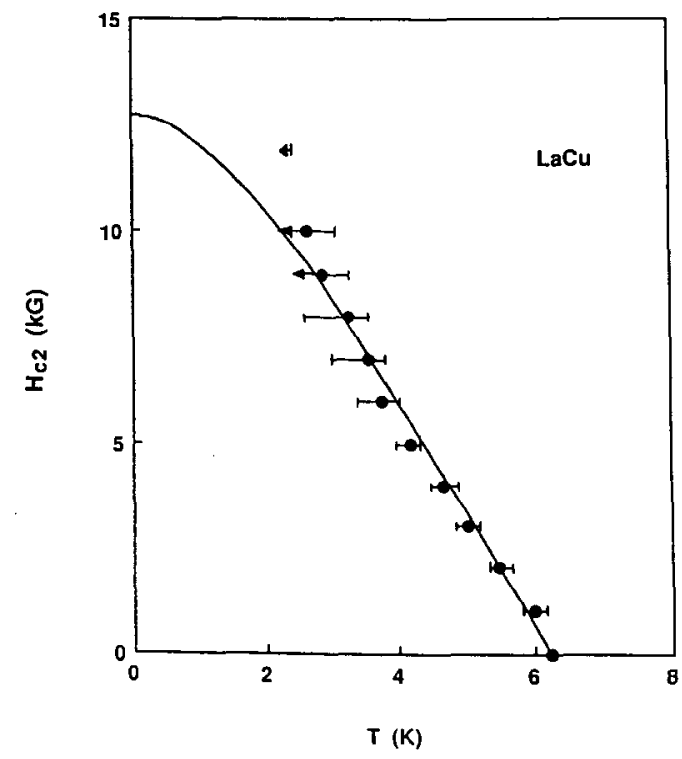

Fig. 3. The upper critical magnetic field $\mathrm{H}_{\mathrm{c} 2}$ as a function of temperature $\mathrm{T}$ for $\mathrm{LaCu}$. The solid circles represent data for $T_{c}(50 \%)$, the horizontal bars represent the transition widths, and the arrows at high fields indicate the transition is not complete at $\mathrm{T} \approx 2.5 \mathrm{~K}$. The $\mathrm{H}_{\mathrm{c} 2}(\mathrm{~T})$ curve calculated from the WHHM theory are also plotted.

The value of the transport mean free path $\mathrm{l}_{t \mathrm{t}}$ for $\mathrm{LaCu}$ can be estimated from its normal state resistivity at low temperatures. The extrapolated zero temperature resistivity $\rho_{0}$ for this sample is estimated to be $1.33 \mu \Omega-\mathrm{cm}$. From this, $1_{t r}$ can be calculated from the formula ${ }^{22} 1_{t r}=(1.27 \mathrm{x}$ $\left.10^{4}\right) / \rho_{0}(Z / \Omega)^{1 / 3}$, where $1_{\mathbb{u}}$ is in $\mathrm{cm}, \rho_{0}$ is in $\Omega-\mathrm{cm}$ and $(Z / \Omega)$ is the density of the conduction electrons. As a rough approximation, we assume that the conduction electrons are contributed by the trivalent $\mathrm{La}$ atoms so that $Z=12$ since there four $\mathrm{LaCu}$ formula units per unit cell. From the lattice parameters for $\mathrm{LaCu}$, we find $\Omega=1.99 \times 10^{22} \mathrm{~cm}^{3}$. The value of $1_{t r} \approx 810 \AA$ is then obtained. Since $\xi_{0} \ll 1_{t r}$, we suggest that $\mathrm{LaCu}$ is a clean superconductor.

The conduction electron effective mass $\mathrm{m}^{*}$ for $\mathrm{LaCu}$ can be estimated from the equation $23 \mathrm{~m}^{*}=\mathrm{h}^{2} \mathrm{k}_{\mathrm{F}} \gamma / 4 \pi^{4}(\mathrm{Z} / \Omega) \mathrm{k}_{\mathrm{B}}{ }^{2}$. Here $\gamma$ is the electronic specific heat coefficient at low temperatures which has a value of $10 \mathrm{~mJ} / \mathrm{mole}-\mathrm{K}^{2}$ as obtained from the $C(T)$ measurement and $\mathrm{KF}_{F}$ is the Fermi wave vector which, with the assumption of a spherical Fermi surface in the sample, can be calculated from the relation $\mathrm{kF}$ $=\left(3 \pi^{2} \mathrm{Z} / \Omega\right)^{1 / 3}$ and yields a value of $1.21 \times 10^{8} \mathrm{~cm}^{-1}$. From this, we then obtain $m^{*} \approx 4 m_{e}$, where $m_{e}$ is the free-electron mass.

The curve calculated from the Werthamer, Helfand, Hohenberg, and Maki (WHHM) theory 24,25 of $\mathrm{H}_{\mathrm{c2}}$ (T) for the conventional type II superconductor using the experimental values of the initial slope $\left(-\mathrm{dH}_{\mathrm{c} 2} / \mathrm{dT}\right) \mathrm{Tc} 0$ and $\mathrm{T}_{\mathrm{c} 0}$ for the minimum $\left(\lambda_{\mathrm{so}}=\infty\right)$ paramagnetic limit, where $\lambda_{\text {so }}$ is the spin orbit scattering parameter, are also plotted in Fig. 3. The calculated $\mathrm{H}_{\mathrm{c} 2}(\mathrm{~T})$ curve fits the experimental data quite well in the whole temperature range. The calculated upper critical field at zero temperature $\mathrm{H}_{\mathrm{c} 2}(0)$ is $22.6 \mathrm{kG}$.

In summary, we have performed the $\mathrm{H}_{\mathrm{c} 2}(\mathrm{~T})$ and $\mathrm{C}(\mathrm{T})$ studies of $\mathrm{LaCu}$. The initial slope $\left(-\mathrm{dH}_{\mathrm{c} 2} / \mathrm{dT}\right)_{\mathrm{Tc} 0}$ of this compound is of $2.97 \mathrm{kG} / \mathrm{G}$. The calculated $\mathrm{H}_{\mathrm{c} 2}(\mathrm{~T})$ curve using the WHHM theory can fit the experimental data in the whole temperature range quite well. The obtained value of $\mathrm{l}_{\mathrm{tr}}$ is $\approx 162 \dot{A}$, which is much than the value of $\xi_{0}$ and implies clean superconductivity in this system. The Debye temperature of this system is about $148 \mathrm{~K}$ and the effective mass of the conduction electrons is about $4 \mathrm{~m}_{\mathrm{e}}$ as estimated from the $C(T)$ data. This work was supported by the $R$. $O$. C. National Science Council under Grant No. NSC 810208-M002-045.

\section{References}

1. B. T. Matthias, H. Suhl, and E. Corenzwit, Phys. Rev. Lett. 1, 92 (1958).

2. R. A. Hein, R. L. Falge Jr., B. T. Matthias, and E. Corenzwit, Phys. Rev. Lett. 2, 500 (1959).

3. M. B. Maple, Physics Letters, 26A, 513 (1968).

4. B. T. Matthias, H. Suhl, and Corenzwit, Phys. Rev. Lett. 1.449 (1958).

5. W. Wilhelm and B. Hillenbrand, J. Phys. Chem. Solids, 31, 559 (1970).

6. J. W. Lynn, D. E. Moncton, L. Passell, and W. Thomlinson, Phys. Rev. B21, 70 (1980).

7. T. F. Jones, J. F. Kwak, E. P. Chock, and P. M. Chaikin, Solid State Commun. 27, 209 (1978).

8. R. Chevrel, M. Sergent, and J. Prigent, J. Solid State Chem. 3, 515 (1971).
9. B. T. Matthias, M. Marezio, E. Corenzwit, A. S. Cooper, and H. Barz, Science 175, 1465 (1972).

10. J. M. Vandenberg and B. T. Matthias, Proc. Natl. Acad. Sci. 24, 1336 (1977).

11. B. T. Matthias, E. Corenzwit, J. M. Vandenberg, and H. Barz, Proc. Natl. Acad. Sci. 74, 1334 (1977).

12. Topics in Current Physics, Vol. 32, eds. $\varnothing$. Fischer and M. B. Maple (Springer-Verlag, New York, 1982).

13. Topics in Current Physics, Vol. 34, eds. M. B. Maple and $\varnothing$. Fischer (Springer-Verlag, New York, 1982).

14. A. C. Larson and D. T. Cromer, Acta. Cryst. 14, 545 (1961).

15. R. E. Walline and W. E. Wallace, J. Chem. Phys. 42, 604 (1965). 
16. A. Iandelli and A. Palenzona, J. Less-Common Metals, 25, 333 (1971).

17. T. F. Smith and H. L. Luo, J. Phys. Chem. Solids. 28, 569 (1967).

18. E. Bauer, Advance in Physics. 40, 417 (1991).

19. J. W. Chen, J. Magn. Mag. Mat. in press.

20. R. R. Hake, Appl. Phys. Lett. 10, 186 (1967).

21. For example, see M. Tinkham, "Introduction to Superconductivity" (McGraw-Hill, New York, 1975).
22. T. P. Orlando, E. J. McNiff, S. Foner, and M. R. Beasley, Phys. Rev. B19, 4545 (1979).

23. For example, see C. Kittel, "Introduction to Solid State Physics", 6th edition, (John Wiley \& Sons, New York, 1986).

24. N. R. Werthamer, E. Helfand, and P. C. Hohenberg, Phys. Rev. 147, 295 (1966).

25. K. Maki, Phys. Rev. 148, 392 (1966). 